\title{
Can nasal Staphylococcus aureus screening and decolonization prior to elective total joint arthroplasty reduce surgical site and prosthesis-related infections? A systematic review and meta-analysis
}

Xingyang Zhu ${ }^{1,2+}$, Xiaobo Sun ${ }^{1+}$, Yuqing Zeng ${ }^{1}$, Wenjun Feng ${ }^{3}$, Jie $\mathrm{Li}^{3}$, Jianchun Zeng ${ }^{3}$ and Yirong Zeng ${ }^{3^{*}}$

\begin{abstract}
Background: Nasal Staphylococcus aureus (S. aureus) screening and decolonization has been widely used to reduce surgical site infections (SSIs) prior to total knee and hip arthroplasty (TKA and THA). However, it remains considerably controversial. The aim of this study was to ascertain whether this scheme could reduce SSIs and periprosthetic joint infections (PJIs) following elective primary total joint arthroplasty (TJA).

Methods: A systematic search was performed in MEDLINE, Embase, and the Cochrane Library until October, 2019. Outcomes of interest included SSI, PJI, superficial infection, and different bacterial species that caused infections. Data from eligible studies were then extracted and synthesized. Pooled odds ratios (OR) and 95\% confidence intervals (Cls) were calculated. We also performed additional analyses to evaluate whether there were differences in postoperative SSIs caused by S. aureus or other bacteria.

Results: Nine studies were included in our meta-analysis. The pooled data elucidated that nasal S. aureus screening and decolonization dramatically mitigated the risk of SSI, PJI, and superficial infection compared to nondecolonization group. The analysis of bacterial species causing infection also showed that the $S$. aureus infections postoperative were significantly decreased in the decolonization group. However, there was no statistical difference in the SSI caused by other bacteria between the two groups.
\end{abstract}

Conclusion: S. aureus screening and decolonization prior to elective primary THA and TKA could significantly decrease the risk of SSI and PJI. However, more robust studies are needed to further evaluate the impact of $S$. aureus screening and decolonization on infection risk after TJA.

Keywords: Staphylococcus aureus, Surgical site infection, Periprosthetic joint infection, MRSA, Screening, decolonization

\footnotetext{
* Correspondence: 15713973727@126.com

†Xingyang Zhu and Xiaobo Sun contributed equally to this work.

${ }^{3}$ Department of Orthopaedics, The First Affiliated Hospital of Guangzhou

University of Chinese Medicine, Jichang Road 16\#, District Baiyun,

Guangzhou 510405, Guangdong, China

Full list of author information is available at the end of the article
}

(c) The Author(s). 2020 Open Access This article is distributed under the terms of the Creative Commons Attribution 4.0 International License (http://creativecommons.org/licenses/by/4.0/), which permits unrestricted use, distribution, and reproduction in any medium, provided you give appropriate credit to the original author(s) and the source, provide a link to the Creative Commons license, and indicate if changes were made. The Creative Commons Public Domain Dedication waiver (http://creativecommons.org/publicdomain/zero/1.0/) applies to the data made available in this article, unless otherwise stated. 


\section{Introduction}

Periprosthetic joint infection (PJI) is a devastating complication following total joint arthroplasty (TJA) and accounts for the majority of postoperative revisions $[1,2]$. Meanwhile, it brings a huge economic burden to the healthcare system each year [3-5]. Therefore, the prevention of PJI cannot be overemphasized. Recently, more and more surgeons have emphasized the importance of improving modifiable risk factors preoperatively [6-9]. The nasal Staphylococcus aureus colonization is generally considered to be one of the modifiable risk factors [6].

Some studies showed that the routine implementation of nasal screening and selective decolonization could significantly mitigate the risk of surgical site infection (SSI) following TJA [10-13]. However, it is still controversial, because several studies suggested the opposite conclusion $[14,15]$.

Therefore, the purpose of this systematic review and meta-analysis was to quantitatively evaluate (1) was there any association between the nasal carrying of $S$. aureus and the high rate of SSI or PJI after primary elective total hip and total knee arthroplasty (THA and TKA), (2) could preoperative nasal $S$. aureus screening and decolonization reduce the rate of SSI and PJI, and (3) was the routine $S$. aureus screening necessary before TJA.

\section{Methods}

This study was conducted strictly according to the Preferred Reporting Items for Systematic Reviews and Meta-Analyses statement [16]. Before the start of literature searches, the research protocol was determined by all coauthors.

\section{Search strategy}

Following the PICOS methodology, two authors (Xingyang Zhu and Xiaobo Sun) developed the search strategies with the assistance of an experienced librarian. The last database search was performed on October 18, 2019 with comprehensive strategies, including both Medical Subject Headings and keywords, for the following electronic databases: MEDLINE (PubMed), EMBASE (Elsevier platform), and COCHRANE CENTRAL (through the Cochrane-Library). When searching, we had no restrictions on the language and publication date of the articles to maximize the sensitivity. Additionally, reference lists of relevant articles were also screened to identify additional studies. See Appendix 1 for the full and detailed search strategy.

\section{Inclusion and exclusion criteria}

Two independent authors assessed the titles or abstracts or full text of the final search results. In case of any controversy that arose between the two reviewers (Xingyang Zhu and Xiaobo Sun) regarding eligibility, an agreement could be reached through discussion. If no consensus was achieved, the final decision was made by the third author (Yirong Zeng).

The inclusion criteria were listed as follows: (1) all surgical procedures were primary THA or TKA; (2) data detailing SSI rate in patients with or without preoperative $S$. aureus screening and decolonization after primary THA or TKA were complete; (3) sufficient data were provided for calculating pooled odds ratios (OR) with a 95\% confidence interval (CI); (4) specific information on the measures of $S$. aureus screening and decolonization as well as the antibiotic application perioperatively was available.

The following exclusion criteria were used: (1) those studies that the data of interest were incomplete; (2)patients were treated with emergency joint replacements (e.g., hip arthroplasty for femoral neck fracture) or revision surgeries; (3) research contained other orthopedic surgeries; (4) full manuscript was not available; (5) reduplicative studies of the same patients in different periods; (6) reviews, commentaries, editorials, and letters were excluded; (7) languages were not accessible to authors.

\section{Study quality assessment}

The methodological study quality was assessed using the Newcastle-Ottawa Scale (NOS), a validated tool suitable for cohort and case-control study [17]. Two reviewers assessed the studies independently, resolved divergences through discussions or reached consensus with the third author.

\section{Data extraction}

Pertinent data were extracted by two reviewers independently from all eligible studies using a standardized data collection form, including the following variables: author, year of publication, country, type of study, number of TJR, type of arthroplasty (THA, TKA, or both), the methods of $S$. aureus screening and decolonization, application of perioperative antibiotics, definition of PJI, and any wound complications, number of SSI (PJI and superficial infection), and infection rates of $S$. aureus and other bacteria. For research lacking the results we needed, we had contacted the author(s) via email for more information.

The primary outcome of this research was SSI. However, different studies followed various definitions of SSI and PJI. Therefore, similar to Bedard et al. [7], "any wound complications" and "PJI" were used to pool end points of reported infection. "Any wound complications" was defined as any postoperative wound complications reported in the included studies, while PJI was defined as any deep infection involving prosthesis. The secondary outcome was the infection rate of $S$. aureus and other bacteria between the two groups. 


\section{Statistical analysis}

The OR and associated 95\% CI were used to perform estimates for dichotomous variables, while the mean \pm standard deviation was used for continuous variables. Only research that contained both OR and CI could be included in the analysis. $P$ values $<0.05$ were considered to be statistical significance.

The $I 2$ and $p$ value were used to evaluate the statistical heterogeneity across studies. If the heterogeneity test indicated the $p$ values $>.1$ or $I^{2}<50 \%$, the fixed effect model would be applied. On the contrary, if the heterogeneity test expressed the $p$ values $\leq .1$ or $I^{2} \geq 50 \%$, the random effect model would be used. If necessary, a sensitivity analysis was conducted to assess the stability of the results. If data were available, subgroup analysis was also conducted to get more specific conclusions. In addition, forest plots were used to depict the results of each study and evaluate pooled estimates respectively, and the funnel plots were used to evaluate publication bias. All statistical analyses were performed through Review Manager (version 5.3.5 for Windows; The Cochrane Collaboration, The Nordic Cochrane Center, Copenhagen, 2014).

\section{Results}

\section{Study selection}

A total of 164 potentially relevant articles were identified from the three electronic databases through systematic search. Forty-five duplicates were deleted by citation management software and manual review of records. After two authors reviewed the titles and abstracts, 73 irrelevant citations were removed. The remaining 46 fulltext papers were then retrieved for a more detailed analysis, of which 37 papers were excluded for several reasons, such as irrelevant research $(n=18)$, lacking raw date $(n=4)$ [18-21], containing revision or other

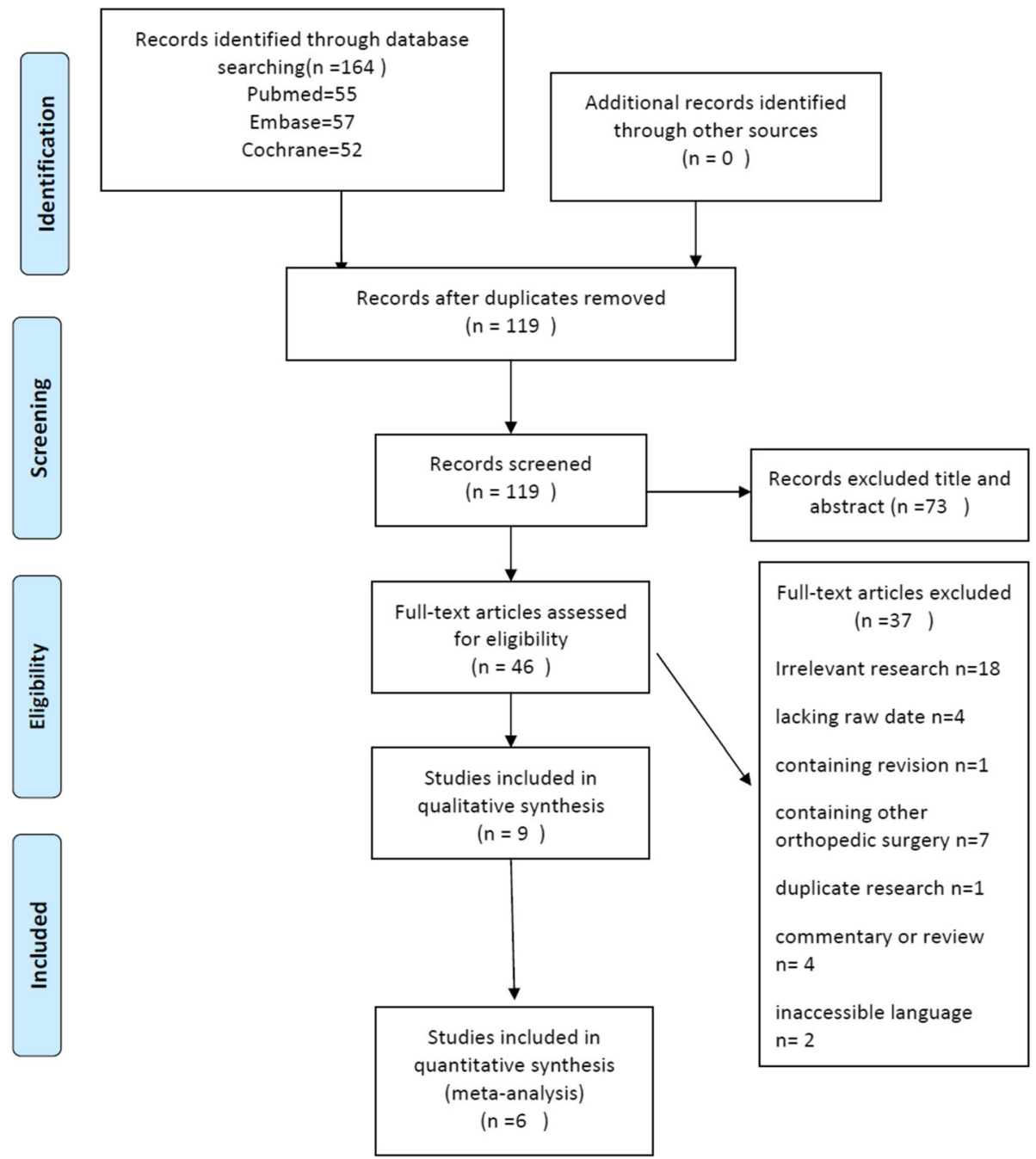

Fig. 1 Summary of the evidence search and selection process 


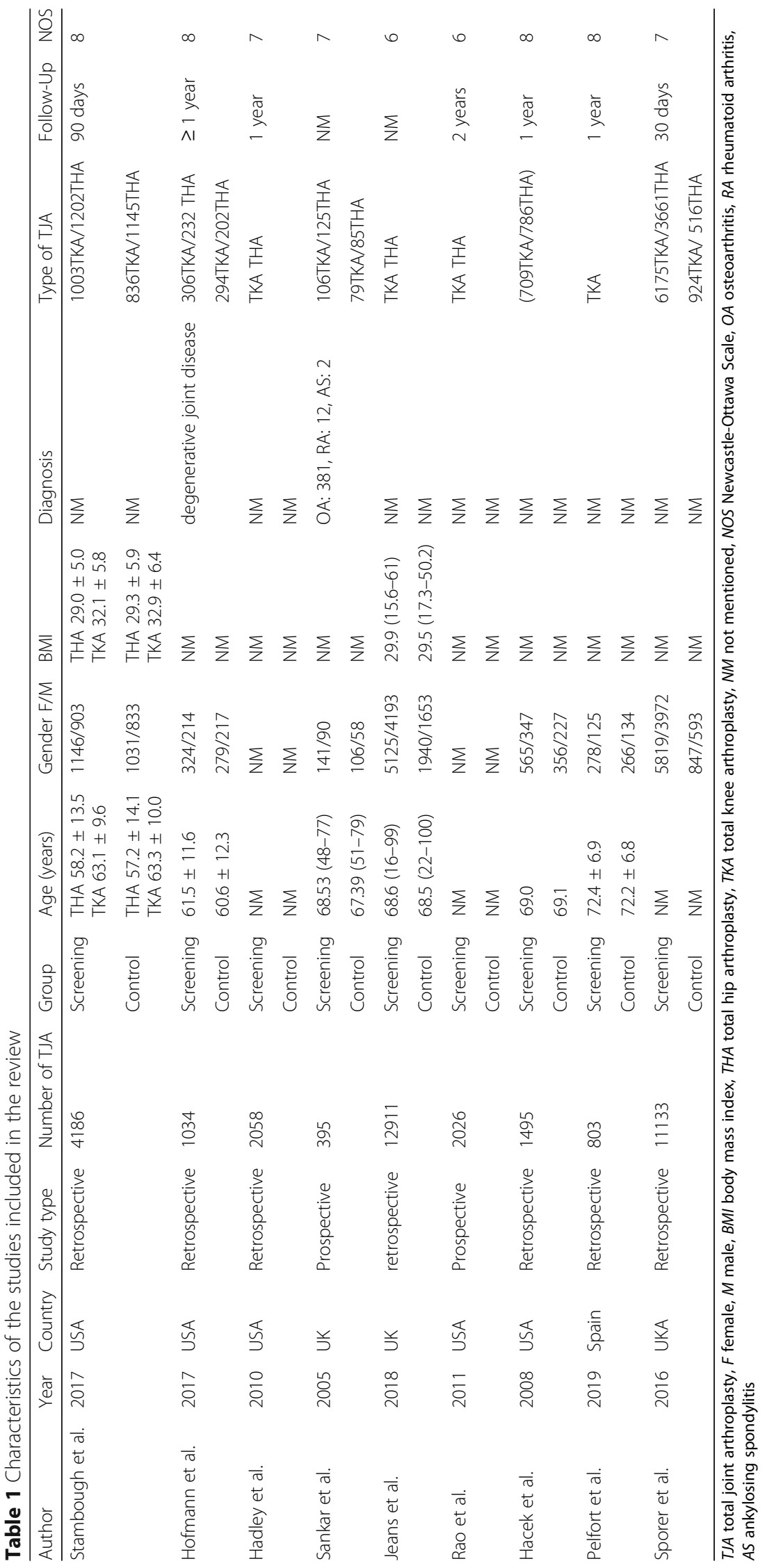




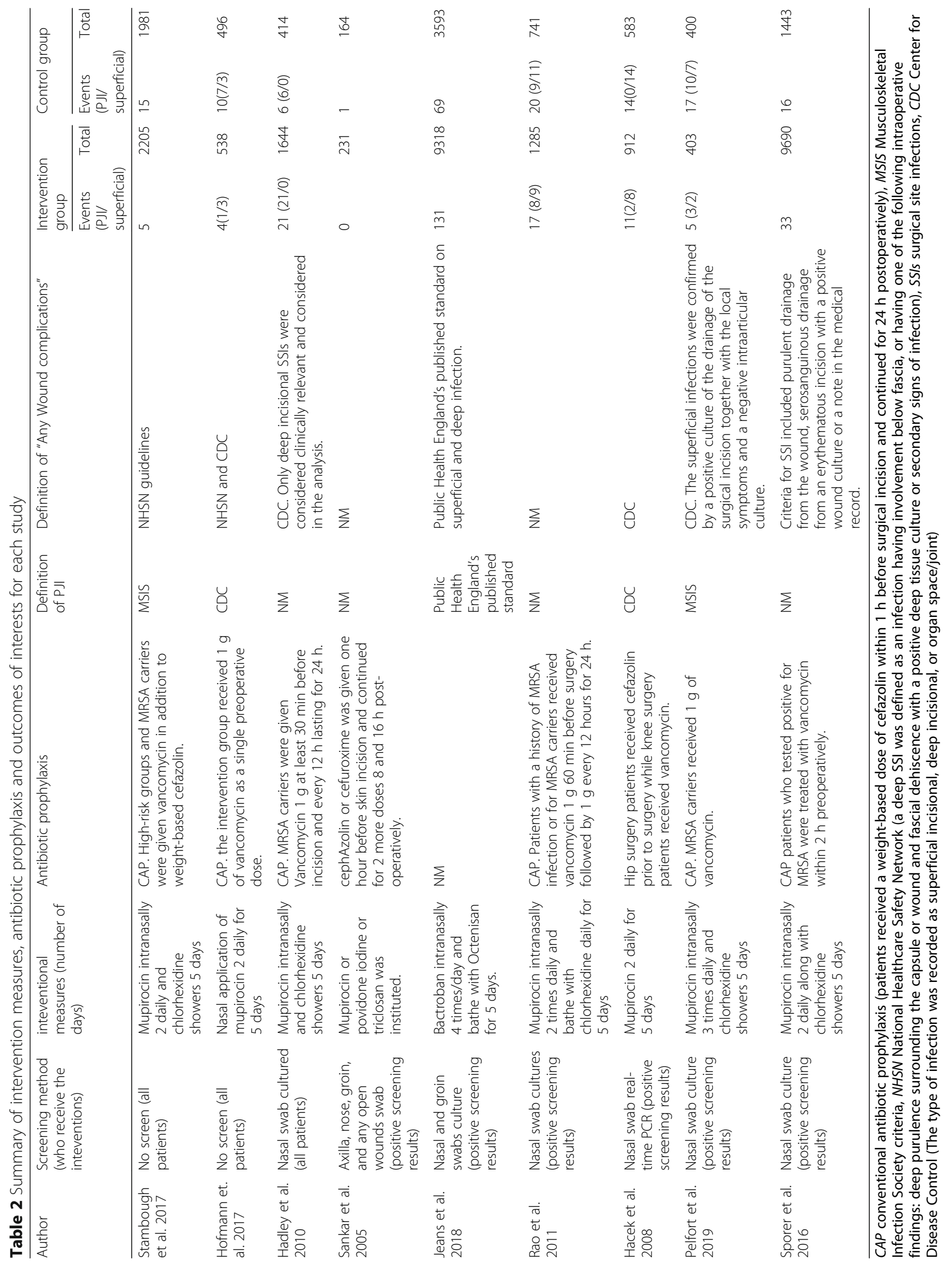


orthopedic surgery $(n=8)$ [14, 22-28], duplicate research $(n=1)$ [29], commentary or review $(n=4)$ [2033 , and inaccessible language $(n=2)$ [34, 35]. Finally, a total of nine studies were included in this research [4, 10-13, 36-39]. Six of them were performed multivariate analysis [10-13, 37, 38], and the remaining three studies were given a descriptive analysis of findings because of slightly different intervention methods $[4,36,39]$. The study selection process throughout the different phases is shown in Fig. 1.

\section{Study characteristics and quality}

A total of 36041 joint replacements were included in the nine studies, of which 26226 were divided into S. aureus screening and decolonization group, and the remaining 9815 were the control group. Except for one study that only included TKA [10], all other studies included TKA and THA [4, 11-13, 36-39]. Among the nine studies, six were from the USA $[4,11-13,37,38]$, two from the UK [36, 39], and one from Spain [10]. The longest follow-up period in these studies was 2 years [11], and the shortest follow-up time was 30 days [38], while it was difficult to find specific follow-up time in two studies $[36,39]$. We tried to contact the authors, but failed. So we performed a statistical description of these two studies instead of the combination of effect sizes. Of the included studies, there were two prospective studies and seven retrospective studies with a score of 6 to 8 stars according to the NOS. Overall, these studies were of moderate to high quality. The detailed study qualities are shown in Appendix 2. A summary of the general characteristics, intervention measures, antibiotic prophylaxis, and outcomes of interests of all included studies in this review is displayed in Tables 1 and 2 .

\section{Strategies of antibiotic prophylaxis}

Different studies had various strategies for antibiotic prophylaxis. The most common methods of antibiotic prophylaxis were as follows: patients received a weightbased dose of cefazolin within 30-60 min before surgical incision and continued for $24 \mathrm{~h}$ postoperatively in both groups, while methicillin-resistant $S$. aureus (MRSA) carriers were treated with vancomycin $1 \mathrm{~g}$ at least 30 min before incision and every $12 \mathrm{~h}$ lasting for $24 \mathrm{~h}$ in screening group. However, in the study performed by Hofmann et al. [37], all patients received $1 \mathrm{~g}$ of vancomycin preoperative in the screening group. In another study of Hacek et al. [13], hip surgery patients were treated with cefazolin preoperatively while knee surgery patients were given vancomycin in the screening group.

\section{Definition of SSI}

The majority of studies included in this review used the diagnostic criteria of Center for Disease Control (CDC) and/or National Healthcare Safety Network (NHSN) for any wound complications $[4,10,12,13,37]$, while Jeans et al. [36] used the Public Health England's published standard, Sporer et al. [38] diagnosed SSI based on clinical symptoms, positive wound culture, and a note in the medical record, while Rao et al. [11] and Sankar et al. [39] did not give a specific definition.

\section{S. aureus decolonization and SSIs}

Six studies [10-13, 36-38] that assessed 18549 TJA reported postoperative SSI. The pooled analyses demonstrated that $S$. aureus screening and decolonization prior to surgery decreased the risk of subsequent SSI $(\mathrm{OR}=$ $0.43,95 \%$ CI 0.31 to $0.59, I^{2}=0 \%, p<0.001$; Fig. 2). The funnel plots indicated no obvious publication bias (Fig. 3).

Due to varying intervention methods used in the original studies, three studies could not be included in pooled data [4, 36, 39]. Stambough et al. [4] found that both the overall SSI rate (5 vs. 15 cases, $p=.013$ ) and SSI caused by $S$. aureus (2 vs. $10, p=.01$ ) were significantly decreased; however, compared with traditional methods, the patients in the screening group were all given vancomycin prior to surgery, and all patients in the control group were also screened for $S$. aureus colonization and selectively treated with mupirocin preoperatively. Sankara et al. [39] found that there was a significant reduction in hospital-acquired infection rate following the screening for MRSA prior to TJR. Jeans et al. [36] also demonstrated that screening and eradication of methicillin-sensitive $S$. aureus (MSSA) could not only effectively reduce the incidence of MSSA PJI but also save a lot of costs. Nevertheless, except for nasal

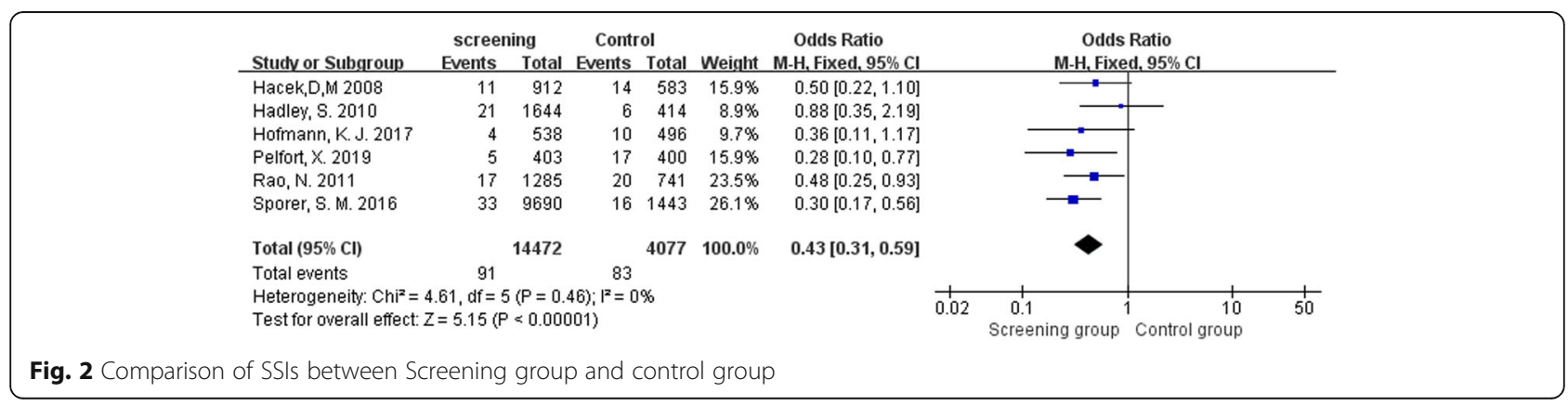




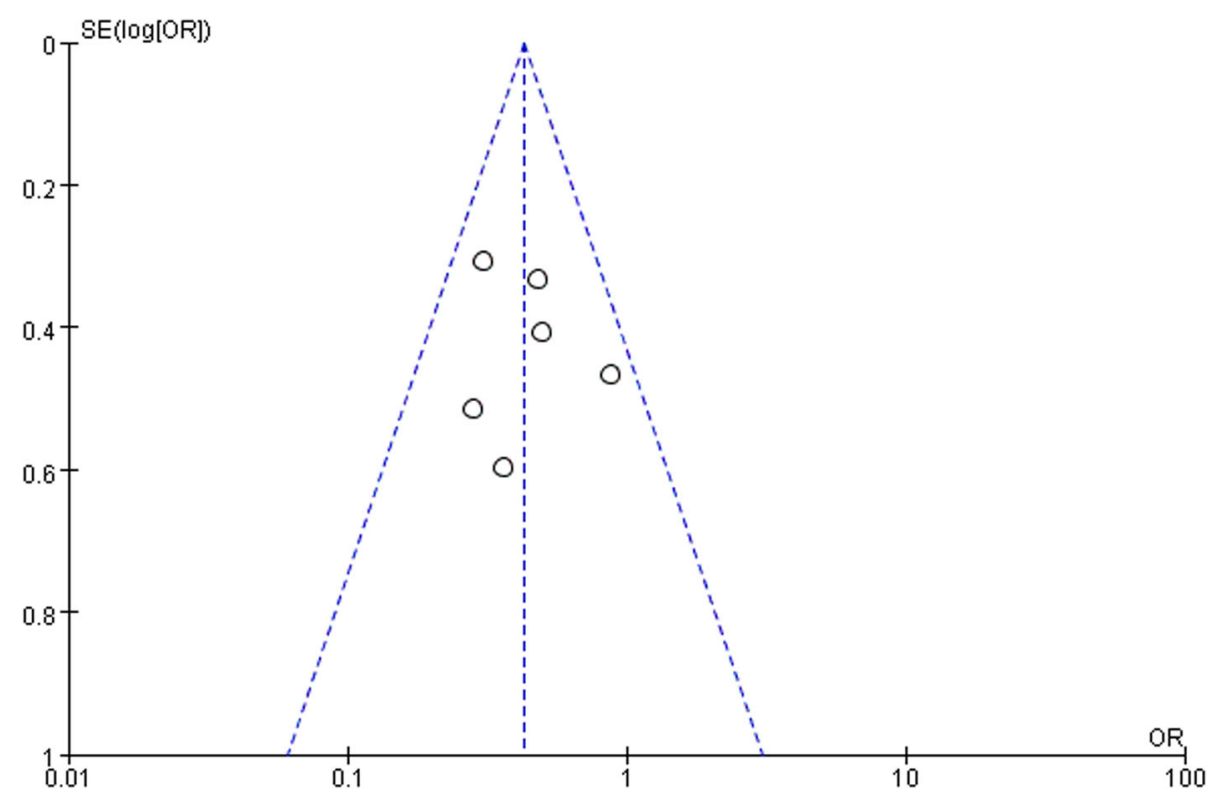

Fig. 3 Funnel plot illustrating a meta-analysis of the SSIs. SE standard error, OR odds ratio

screening of $S$. aureus colonization, these two studies also screened groin and/or armpit [36, 39].

Only four studies provided relevant information about deep and superficial infection [10, 11, 13, 37]. Infection category was not distinguished in the study by Sporer et al. [38], while only deep infection was included in the analysis of Hadley et al. [12]. To assess whether there was a difference in the effects of $S$. aureus screening and decolonization on PJI and superficial infection, we performed a subgroup analysis. The pooled analyses demonstrated significant statistical differences between the two groups both in PJI (OR $=0.40,95 \%$ CI 0.21 to $0.77, I^{2}=$ $11 \%, p=0.006$; Fig. 4) and superficial infection ( $\mathrm{OR}=$ $0.43,95 \%$ CI 0.251 to $0.73, I^{2}=0 \%, p=0.002$; Fig. 5).

Six studies [10-13, 37, 38] including 18549 TJR assessed postoperative SSI caused by $S$. aureus or other bacteria. Table 3 presents detailed information on the distribution of S. aureus and other bacteria in SSI with or without S. aureus screening and decolonization. The pooled data showed a significant statistical difference in postoperative $S$. aureus infection between the two groups $(\mathrm{OR}=0.20,95 \% \mathrm{CI} 0.11$ to $0.34, I^{2}=30 \%, p<0.001$; Fig. 6). However, there was no statistical difference in SSI caused by other bacteria $(\mathrm{OR}=$ $0.73,95 \%$ CI 0.48 to $1.12, I^{2}=0 \%, p=0.15$; Fig. 7 ).

\section{Discussion}

This systematic review and meta-analysis included nine independent studies that analyzed 36041 cases of arthroplasty and directly assessed the effectiveness of $S$. aureus decolonization and nondecolonization in SSI following primary THA and TKA procedures. The pooled analyses indicated that $S$. aureus screening and decolonization reduced the SSI (both PJI and superficial infection). And further research suggested a decrease in SSI caused by $S$. aureus, while there was no difference in SSI caused by other bacteria between the two groups.

The nasal cavity is one of the most common sites for $S$. aureus colonization. One study revealed that the nasal colonization rate of $S$. aureus was $22.2 \%$ and that of MRSA was $0.8 \%$ [15]. Another study also showed that colonization rate of MSSA was $22.6 \%$ and that of MSRA was $4.4 \%$ [40]. It is generally believed that $S$. aureus in

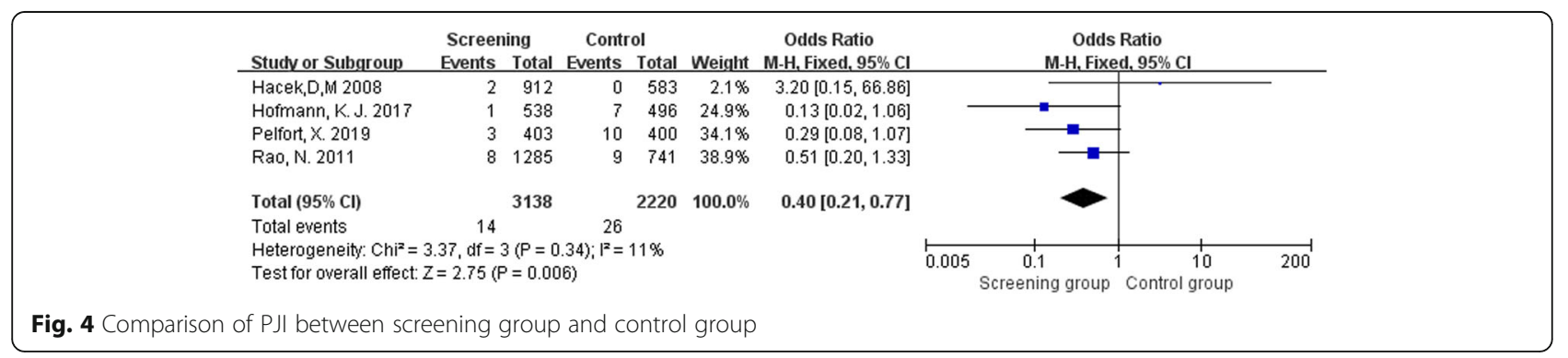




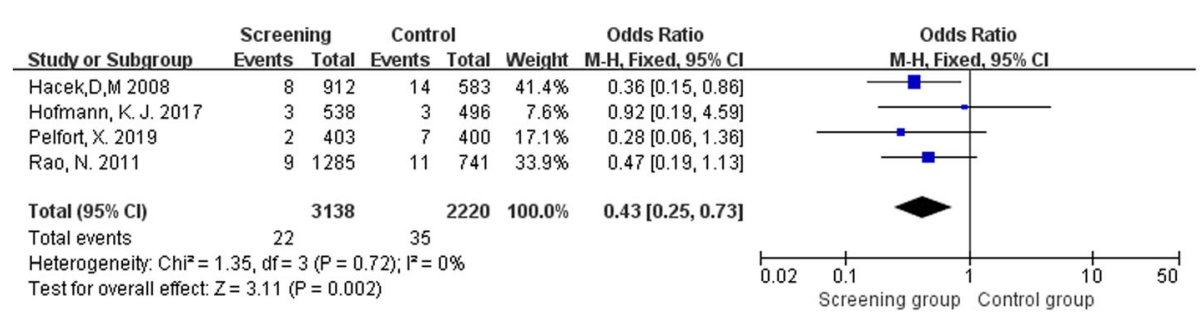

Fig. 5 Comparison of superficial infection between Screening group and control group

the nasal cavity is one of the potential sources of bacterial seeding for SSI. The methods of S. aureus screening mainly include bacterial culture and real-time polymerase chain reaction (RT-PCR).

Currently, the common method for $S$. aureus decolonization is that topical application of mupirocin twice daily to both nares accompany with or without chlorhexidine showers or skin wipes daily for 5 days prior to the TJA [41]. However, several studies implemented an extensive decolonization program, regardless of whether $S$. aureus was positive or not $[4,12,37]$, which may be considered an abuse of antibiotics and may lead to an increased risk of bacterial resistance. Therefore, it may be more reasonable to perform selective decolonization programs for only screened positive patients.

Conventional application of this regimen to patients with positive $S$. aureus preoperative has shown good short-term results in improving decolonization rates [4]. However, its long-term efficacy is uncertain. Many studies showed that a significant number of patients would remain positive for $S$. aureus after surgery $[42,43]$. For example, a study revealed that 33\% (19 of 58) of patients still performed positive for $S$. aureus colonization at 3-30 months after surgery despite preoperative decolonization [44]. Another recent study also showed that the screening and decolonization regimen did significantly reduce the number of nasal carriage of MRSA, but the study also showed that about $20 \%$ of patients might remain colonized by MRSA despite a decolonization protocol in patients undergoing Elective TJA [28]. This means that a decolonization treatment could not guarantee that patients will keep decolonized in the future, which requires further research, because it is still unclear whether the risk of late infection in this population is higher [44].

So far, whether the $S$. aureus decolonization program could reduce the SSI in patients undergoing primary TJA is still controversial. Numerous studies showed that SSI could be decreased by an institutional nasal screening and decolonization protocol for patients before elective TJA regardless of carriers of either MSSA or MRSA [38, 40]. For instance, Sporer et al. [38] demonstrated a $69 \%$ reduction in the prevalence of SSI after the use of screening and decolonization program. Similar to this study, Kim et al. [40] also reported a 59\% reduction in SSI after implementing a similar screening protocol, and they found that MRSA carriers had a higher rate of SSI than that of non-carriers $(0.97 \%$ vs. $0.19 \%)$. Therefore, nasal carriage of $S$. aureus has been considered as an independent risk factor for SSI and PJI. Conversely, several recent studies demonstrated that nasal decolonization protocol prior to elective TJA could not decrease the incidence of SSI $[14,15,28]$. After comparing the infection rates of colonized and noncolonized patients, Ramos et al. [14] discovered that the risk of infection in decolonized patients could not be decreased to the baseline level of nondecolonized patients. A prospective randomized trial also demonstrated that there was no significant difference between treated and untreated carriers and that most of $S$. aureus PJI occurred in non-carriers, so the authors believed that there was no clear benefit in screening and/or decolonizing carriers before TJA [15].

Table 3 Distribution of S. aureus and other bacteria in SSIS

\begin{tabular}{|c|c|c|c|c|}
\hline \multirow[t]{2}{*}{ Author } & \multicolumn{2}{|l|}{ Screening group } & \multicolumn{2}{|l|}{ Control group } \\
\hline & S. aureus/other bacterial infection & Total & S. aureus/other bacterial infection & Total \\
\hline Hofmann et al. 2017 & $1 / 3$ & 538 & $3 / 7$ & 496 \\
\hline Hadley et al. 2010 & $3 / 18$ & 1644 & $1 / 5$ & 414 \\
\hline Rao et al. 2011 & $0 / 17$ & 1285 & $12 / 8$ & 741 \\
\hline Hacek et al. 2008 & $7 / 4$ & 912 & $10 / 4$ & 583 \\
\hline Pelfort et al. 2019 & $1 / 4$ & 403 & $8 / 9$ & 400 \\
\hline Sporer et al. 2016 & $11 / 22$ & 9690 & $11 / 5$ & 1443 \\
\hline
\end{tabular}




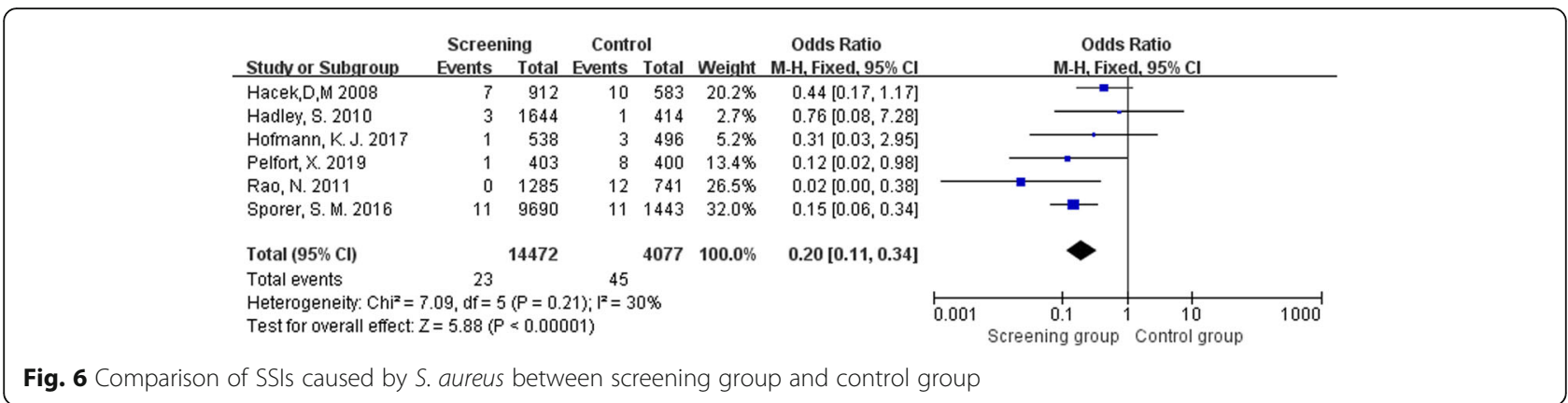

Based on the included studies, our pooled data showed that $S$. aureus screening and decolonization dramatically reduced the incidence of SSI. However, it should be noted that in these studies, patients screened positive for MRSA also received vancomycin as a standard perioperative antibiotic prophylaxis, so it did not rule out that the decrease of infection rate might be caused by the use of vancomycin, which needs to be further studied. Additionally, Jeans et al. [36] reported that MSSA screening and decolonization might be more effective in decreasing overall infection rate in hips than in knees. Unfortunately, due to insufficient data in this area, we are unable to conduct relevant analysis.

To the best of our knowledge, this is the most comprehensive meta-analysis examining the association of $S$. aureus screening and decolonization with SSI after TKA and THA. We are aware of previous meta-analyses on this topic $[30,45,46]$. Studies performed by Levy et al. [45] and Chen et al. [46] contained other orthopedic surgeries, which would lead to a mass of confounding factors. In another very recent paper performed by Sadigursky et al. [30], only four studies were included in the analysis. Compared with these studies, we included some new clinical studies up to 2019. Moreover, we strictly assessed the quality of all selected studies and followed the PRISMA statement in this study.

Although we have designed and implemented the research as perfectly as possible, our meta-analysis still inevitably has several inherent limitations. First, as all systematic reviews, some studies would be ignored because of search strategies. In order to overcome this problem as much as possible, we consulted professional librarians and optimized search strategies continuously during the search process. Second, most of the included studies were retrospective rather than prospective, and follow-up duration of some selected studies was short (about 30-90 days), which might prevent late PJI and SSI from being observed. Third, the diagnostic criteria of PJI and SSI in different studies were not uniform, and preoperative treatment with vancomycin might affect the outcomes for patients screened positive for MRSA. Finally, the data analysis in this paper were performed at the research level rather than at the patient level; therefore, we could not take each patient's physical status, age, gender, diagnoses, body mass index, American society of anesthesiologist (ASA), duration of surgery, nutritional status, and follow-up times into account, all of which might affect the results. Given the above shortcomings, more prospective and long-term follow-up studies are still needed to better understand the impact of the S. aureus screening and decolonization on the incidence of SSI and PJI after TJA.

\section{Conclusions}

In conclusion, the results of this systematic review and meta-analysis suggested that $S$. aureus screening and decolonization prior to elective primary THA and TKA significantly decreased the risk of SSI and PJI. Therefore,

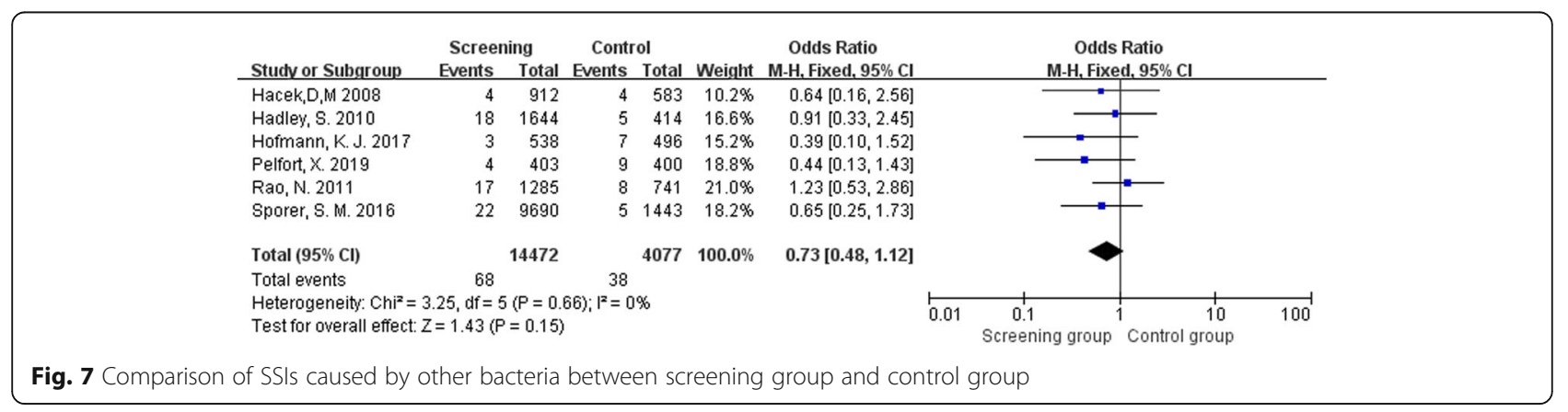


it was our recommendation to implement a standardized universal screening and selective decolonization regimen for all patients undergoing elective TKA and THA. However, these findings were based upon retrospective studies, so lager-scale prospective multicenter studies are needed to evaluate the impact of S. aureus screening and decolonization on SSI and PJI after TJA.

\section{Supplementary information}

Supplementary information accompanies this paper at https://doi.org/10. 1186/s13018-020-01601-0.

Additional file 1. Search Strategy.

Additional file 2. Risk-of-bias assessment for the studies included in the meta-analysis.

\section{Abbreviations}

MRSA: Methicillin-resistant S. aureus; MSSA: Methicillin-sensitive S. aureus; PJl: Periprosthetic joint infection; S. aureus: Staphylococcus aureus; SSI: Surgical site infection; THA: Total hip arthroplasty; TJA: Total joint arthroplasty; TKA: Total knee arthroplasty

\section{Acknowledgments}

One of the first authors (Xingyang Zhu) want to show heartedly thanks to his wife Xiaoyang Si, who provided much help for grammar expression.

\section{Authors' contributions}

Conceptualization: $X Z, Y Z$; literature review and search: $X Z$, XS; data collection: $X Z, X S$; data analysis and interpretation: $Y Z, W F, J L$; manuscript preparation and editing: $X Z, X S$; supervision: JZ, $Y Z$. All authors read and approved the final manuscript.

\section{Funding}

The article received financial support from the High-Level University Construction Project of Guangzhou University of Chinese Medicine(Grant No: A1AFD018171Z11068).

\section{Availability of data and materials}

The authors declare that all the data supporting the findings of this study are available within the article and its supplementary information files.

\section{Ethics approval and consent to participate}

Not applicable.

\section{Consent for publication}

Not applicable.

\section{Competing interests}

The authors declare that they have no competing interests.

\section{Author details}

${ }^{1}$ The First Clinical Medical School, Guangzhou University of Chinese Medicine, Jichang Road 12\#, District Baiyun, Guangzhou, Guangdong, China. ${ }^{2}$ Yichuan People's Hospital, Jiuchang Road 21\#, District Yichuan, Luoyang, Henan, China. ${ }^{3}$ Department of Orthopaedics, The First Affiliated Hospital of Guangzhou University of Chinese Medicine, Jichang Road 16\#, District Baiyun, Guangzhou 510405, Guangdong, China.

Received: 28 December 2019 Accepted: 13 February 2020 Published online: 19 February 2020

\section{References}

1. van Vugt TAG, Arts JJ, Geurts JAP. Antibiotic-loaded polymethylmethacrylate beads and spacers in treatment of orthopedic infections and the role of biofilm formation. Front Microbiol. 2019;10:1626.

2. Kapadia BH, Berg RA, Fritz J, et al. Periprosthetic joint infection. Lancet. 2016; 387(10016):386-94.
3. Williams DM, Miller AO, Henry MW, et al. Cost-effectiveness of Staphylococcus aureus decolonization strategies in high-risk total joint arthroplasty patients. J Arthroplasty. 2017;32(9s):S91-6.

4. Stambough JB, Nam D, Warren DK, et al. Decreased hospital costs and surgical site infection incidence with a universal decolonization protocol in primary total joint arthroplasty. J Arthroplasty. 2017;32(3):728-34.

5. Wang J, Zhu C, Cheng T, et al. A systematic review and meta-analysis of antibiotic-impregnated bone cement use in primary total hip or knee arthroplasty. PLoS One. 2013;8(12):e82745.

6. Alamanda VK, Springer BD. Perioperative and modifiable risk factors for periprosthetic joint infections (PJI) and Recommended Guidelines. Curr Rev Musculoskelet Med. 2018;11(3):325-31.

7. Bedard NA, DeMik DE, Owens JM, et al. Tobacco use and risk of wound complications and periprosthetic joint infection: a systematic review and meta-analysis of primary total joint arthroplasty procedures. J Arthroplasty. 2019;34(2):385-96 e384.

8. Tsantes AG, Papadopoulos DV, Lytras T, et al. Association of malnutrition with periprosthetic joint and surgical site infections after total joint arthroplasty: a systematic review and meta-analysis. J Hosp Infect. 2019; 103(1):69-77.

9. Sousa RJG, Abreu MA, Wouthuyzen-Bakker M, et al. Is routine urinary screening indicated prior to elective total joint arthroplasty? A systematic review and meta-analysis. J Arthroplasty. 2019;34(7):1523-30.

10. Pelfort $X$, Romero A, Brugues $M$, et al. Reduction of periprosthetic Staphylococcus aureus infection by preoperative screening and decolonization of nasal carriers undergoing total knee arthroplasty. Acta Orthop Traumatol Turc. 2019.

11. Rao N, Cannella BA, Crossett LS, et al. Preoperative screening/decolonization for Staphylococcus aureus to prevent orthopedic surgical site infection. prospective cohort study with 2-year follow-up. J Arthroplasty. 2011;26(8):1501-7.

12. Hadley S, Immerman I, Hutzler L, et al. Staphylococcus aureus decolonization protocol decreases surgical site infections for total joint replacement. Arthritis. 2010;2010:924518.

13. Hacek DM, Robb WJ, Paule SM, et al. Staphylococcus aureus nasal decolonization in joint replacement surgery reduces infection. Clin Orthop Relat Res. 2008;466(6):1349-55.

14. Ramos N, Stachel A, Phillips M, et al. Prior Staphylococcus Aureus nasal colonization: a risk factor for surgical site infections following decolonization. J Am Acad Orthop Surg. 2016;24(12):880-5.

15. Sousa RJ, Barreira PM, Leite PT, et al. Preoperative Staphylococcus aureus screening/decolonization protocol before total joint arthroplasty-results of a small prospective randomized trial. J Arthroplasty. 2016;31(1):234-9.

16. Moher D, Liberati A, Tetzlaff J, et al. Preferred reporting items for systematic reviews and meta-analyses: the PRISMA statement. Int J Surg. 2010;8(5):336-41.

17. Stang A. Critical evaluation of the Newcastle-Ottawa scale for the assessment of the quality of nonrandomized studies in meta-analyses. Eur J Epidemiol. 2010;25(9):603-5.

18. Tsang STJ, McHugh MP, Guerendiain D, et al. Evaluation of Staphylococcus aureus eradication therapy in orthopaedic surgery. J Med Microbiol. 2018; 67(6):893-901.

19. Beal S, Long-Brandt W, Tilford M. Prevention of SSIs in total hip and knees using a preoperative antisepsis bundle. Am J Infect Control. 2018;46(6):S80.

20. Thomas $\mathrm{R}$, Koenraadt $\mathrm{K}$, Joosten $\mathrm{P}$, et al. Risk reduction on $\mathrm{PJ}$ I with $\mathrm{S}$. aureus eradication therapy in THA. HIP Int. 2016;26:S42.

21. Rivera $\mathrm{K}$, Smith $\mathrm{RL}$, Rose $\mathrm{L}$, et al. Implementation of a total joint replacement pre-operative skin and nasal decolonization process for the reduction in staphylococcus aureus (SA) and methicillin resistant Staphylococcus aureus (MRSA) Infection. Am J Infect Control. 2016;44(6):S94.

22. Nakamura M, Shimakawa T, Nakano S, et al. Screening for nasal carriage of Staphylococcus aureus among patients scheduled to undergo orthopedic surgery: Incidence of surgical site infection by nasal carriage. J Orthop Sci. 2017;22(4):778-82

23. Schweizer ML, Chiang HY, Septimus E, et al. Association of a bundled intervention with surgical site infections among patients undergoing cardiac, hip, or knee surgery. JAMA. 2015;313(21):2162-71.

24. Campbell KA, Cunningham C, Hasan S, et al. Risk factors for developing Staphylococcus aureus nasal colonization in spine and arthroplasty surgery. Bull Hosp Jt Dis (2013). 2015;73(4):276-81.

25. Pofahl WE, Goettler CE, Ramsey KM, et al. Active surveillance screening of MRSA and eradication of the carrier state decreases surgical-site infections caused by MRSA. J Am Coll Surg. 2009;208(5):981-6. 
26. De Lucas-Villarrubia JC, Lopez-Franco M, Granizo JJ, et al. Strategy to control methicillin-resistant Staphylococcus aureus post-operative infection in orthopaedic surgery. Int Orthop. 2004;28(1):16-20.

27. Wilcox $\mathrm{MH}$, Hall J, Pike $\mathrm{H}$, et al. Use of perioperative mupirocin to prevent methicillin-resistant Staphylococcus aureus (MRSA) orthopaedic surgical site infections. J Hosp Infect. 2003:54(3):196-201.

28. Baratz MD, Hallmark R, Odum SM, et al. Twenty percent of patients may remain colonized with methicillin-resistant Staphylococcus aureus despite a decolonization protocol in patients undergoing elective total joint arthroplasty. Clin Orthop Relat Res. 2015;473(7):2283-90.

29. Rao N, Cannella B, Crossett LS, et al. A preoperative decolonization protocol for staphylococcus aureus prevents orthopaedic infections. Clin Orthop Relat Res. 2008;466(6):1343-8.

30. Sadigursky D, Pires HS, Rios SAC, et al. Prophylaxis with nasal decolonization in patients submitted to total knee and hip arthroplasty: systematic review and meta-analysis. Rev Bras Ortop. 2017;52(6):631-7.

31. Weiser MC, Moucha CS. The current state of screening and decolonization for the prevention of Staphylococcus aureus surgical site infection after total hip and knee arthroplasty. J Bone Joint Surg Am. 2015;97(17):1449-58.

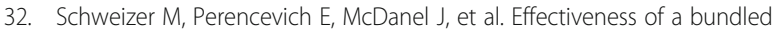
intervention of decolonization and prophylaxis to decrease Gram positive surgical site infections after cardiac or orthopedic surgery: Systematic review and meta-analysis. BMJ (Online). 2013;346:7912.

33. Goyal N, Aggarwal V, Parvizi J. Methicillin-resistant Staphylococcus aureus screening in total joint arthroplasty: a worthwhile endeavor. J Knee Surg. 2012;25(1):37-43.

34. Barbero JM, Romanyk J, Valles A, et al. Decolonization for Staphylococcus aureus carriers in arthroplasty surgery after hip fracture. Rev Esp Quimioter. 2017;30(4):264-8

35. Allende JMB, Romanykcabrera J, Ruiz EM, et al. Eradication of Staphylococcus aureus in carrier patients undergoing joint arthroplasty. Enferm Infecc Microbiol Clin. 2015;33(2):95-100.

36. Jeans $E$, Holleyman $R$, Tate $D$, et al. Methicillin sensitive staphylococcus aureus screening and decolonisation in elective hip and knee arthroplasty. J Arthroplasty. 2018;77(5):405-9.

37. Hofmann K, Hayden BL, Kong Q, et al. Triple prophylaxis for the prevention of surgical site infections in total joint arthroplasty. Current Orthopaedic Practice. 2017;28(1):66-9.

38. Sporer SM, Rogers T, Abella L. Methicillin-resistant and Methicillin-Sensitive Staphylococcus aureus screening and decolonization to reduce surgical site infection in elective total joint arthroplasty. J Arthroplasty. 2016;31(9):144-7.

39. Sankar B, Hopgood P, Bell KM. The role of MRSA screening in jointreplacement surgery. Int Orthop. 2005;29(3):160-3.

40. Kim DH, Spencer M, Davidson SM, et al. Institutional prescreening for detection and eradication of methicillin-resistant Staphylococcus aureus in patients undergoing elective orthopaedic surgery. J Bone Joint Surg Am. 2010;92(9):1820-6.

41. Edmiston CE Jr, Ledeboer NA, Buchan BW, et al. Is Staphylococcal Screening and suppression an effective interventional strategy for reduction of surgical site infection? Surg Infect (Larchmt). 2016;17(2):158-66.

42. Moroski NM, Woolwine S, Schwarzkopf R. Is preoperative Staphylococcal decolonization efficient in total joint arthroplasty. J Arthroplasty. 2015;30(3): 444-6.

43. Chen $A F$, Heyl $A E, X u P Z$, et al. Preoperative decolonization effective at reducing staphylococcal colonization in total joint arthroplasty patients. J Arthroplasty. 2013;28(8 SUPPL):18-20.

44. Economedes DM, Deirmengian GK, Deirmengian CA. Staphylococcus aureus colonization among arthroplasty patients previously treated by a decolonization protocol: a pilot study. Clin Orthop Relat Res. 2013;471(10): 3128-32.

45. Levy PY, Ollivier M, Drancourt M, et al. Relation between nasal carriage of Staphylococcus aureus and surgical site infection in orthopedic surgery: the role of nasal contamination. A systematic literature review and metaanalysis. Orthop Traumatol Surg Res. 2013;99(6):645-51.

46. Chen AF, Wessel CB, Rao N. Staphylococcus aureus screening and decolonization in orthopaedic surgery and reduction of surgical site infections. Clin Orthop Relat Res. 2013;471(7):2383-99.

\section{Publisher's Note}

Springer Nature remains neutral with regard to jurisdictional claims in published maps and institutional affiliations.

Ready to submit your research? Choose BMC and benefit from:

- fast, convenient online submission

- thorough peer review by experienced researchers in your field

- rapid publication on acceptance

- support for research data, including large and complex data types

- gold Open Access which fosters wider collaboration and increased citations

- maximum visibility for your research: over $100 \mathrm{M}$ website views per year

At $\mathrm{BMC}$, research is always in progress.

Learn more biomedcentral.com/submissions 